\title{
MS43-P04 | Mapping Non-Crystalline Nanostructure With LoW-dose Scanning Electron Pair Distribution Function Analysis
}

Laulainen, Joonatan E. M. (Department of Materials Science and Metallurgy, Univesity of Cambridge, Cambridge, GBR); Johnstone, Duncan N. (Department of Materials Science and Metallurgy, University of Cambridge, Cambridge, GBR); Bogachev, Ivan (Department of Materials Science and Metallurgy, University of Cambridge, Cambridge, GBR); Collins, Sean M. (Department of Materials Science and Metallurgy, University of Cambridge, Cambridge, GBR); Longley, Louis (Department of Materials Science and Metallurgy, University of Cambridge, Cambridge, GBR); Bennett, Thomas D. (Department of Materials Science and Metallurgy, University of Cambridge, Cambridge, GBR); Midgley, Paul A. (Department of Materials Science and Metallurgy, University of Cambridge, Cambridge, GBR)

Determining the structure and distribution of non-crystalline phases in complex multi-phase materials is important for understanding novel nanostructured materials such as recently developed glassy MOF composites [1]. Spatially resolved pair distribution function (PDF) analysis, based on scanning electron diffraction (SED) data, provides a route to meet this need [2] as we demonstrate here in application to a glassy ZIF-62: glassy- $60 \mathrm{NaPO}_{3}-40 \mathrm{AIF}_{3}$ composite. Both materials are highly sensitive to electron radiation and it was therefore necessary to perform low-dose ( 10 e/ $\left./ \AA^{2}\right)$ SED experiments, which were enabled using a counting type direct electron detector. The lowdose SED data has an expected low signal-to-noise ratio and we show that independent component analysis (ICA) can be applied to such data to learn physically interpretable PDFs corresponding to the two phases, without the use of prior knowledge. The learnt PDFs are compared with PDFs determined after binning data within phase pure regions to confirm their validity and the spatial localisation determined by ICA is correlated with compositional maps obtained using X-ray energy dispersive spectroscopy (EDS). This analysis shows that ICA combined with lowdose SED can achieve reliable spatially resolved PDF analysis on the nanoscale in beam sensitive materials.

[1] J. M. Tuffnell et al. "Novel Metal-Organic Framework Materials: Blends, Liquids, Glasses and Crystal-Glass Composites." Chemical Communications (2019).

[2] X Mu et al. "Mapping structure and morphology of amorphous organic thin films by 4D-STEM pair distribution function analysis." Microscopy (2019). 